\title{
A Clinical Observation of Thymic Epithelial Tumor Metastatic to Breast
}

\author{
Yu-Wei Deng Yi-Wen Li Wen-Jing Hao Dan Lu \\ Department of Medical Oncology, Second Affiliated Hospital of Harbin Medical University, Harbin, China
}

\section{Established Facts}

- Thymic carcinomas develop local metastases limited to the thorax.

\section{Novel Insights}

- Can there be a hematogenous metachronous metastatic breast lesion originating from thymic carcinoma?

\section{Keywords}

Thymic carcinoma - Metastatic breast cancer .

Hematogenous metastasis

\section{Summary}

Background: Thymic carcinoma is prone to early metastasis and invasion, while its metastasis to the breast is particularly unique. Case Report: We describe a case of thymic epithelial tumor metastatic to the breast fulfilling clinical diagnostic criteria. A 47-year-old female patient diagnosed with stage IV thymic carcinoma and previously treated with chemoradiation was diagnosed with metastatic breast cancer during a periodic review. Color Doppler ultrasonography showed a low-echo real occupancy in the breast. Pathological examination of the breast mass confirmed the diagnosis of thymic carcinoma metastasis. Conclusion: Hematogenous metastasis of thymic carcinoma to the breast is rare but not exceptional, and long-term survival can be expected with appropriate treatment.

(C) 2018 S. Karger GmbH, Freiburg

\section{Introduction}

Thymic carcinomas are rare anterior mediastinal tumors which originate from thymic epithelial cells. According to the pathologic classification developed by the World Health Organization, thymic carcinomas are classified into 5 groups (type $\mathrm{A}, \mathrm{AB}, \mathrm{B} 1, \mathrm{~B} 2$, and B3) by predominate cell type. Thymic carcinomas are usually slow growing with local recurrence rather than metastasis. Sporadic cases with late distant metastasis including lungs, pleura, liver, thyroid, and bones have been reported [1, 2]. However, the majority tend to be local metastases to the chest. Thymus cancer metastasis to the breast is extremely rare. A broad search of the related literature revealed only a few case reports [3]. Although thymus cancer metastatic to the breast is a rare phenomenon, doctors need to be aware of the possibility of this metastasis [4].

\section{Case Report}

In 2013, a 47-year-old woman with no significant medical history was diagnosed with thymic carcinoma. The patient had chest tightness with moderate dull pain without apparent cause, especially after heightened activity. She subsequently presented to our hospital where chest computed tomography (CT) revealed a tumor mass in the anterior mediastinum (fig. 1A). Because of the pres-

\section{KARGER}

() 2018 S. Karger GmbH, Freiburg

Fax +497614520714
Dr. Dan Lu, M.D.

Department of Medical Oncology

Second Affiliated Hospital of Harbin Medical University

Harbin, China

doctorlu1972@163.com 
ence of a mediastinal mass on a chest radiogram, thymoma was suspected to be the underlying cause. The patient underwent a thymus biopsy, and histopathology revealed poorly differentiated squamous cell carcinoma of the thymus with strong expression of cytokeratin $5 / 6$, cytokeratin $8 / 18$, cytokeratin 19 , neuroendocrine markers (CD5, CD117), and anti-oncogene p53 according to immunohistochemical staining (fig. 2A, B). The patient received chemotherapy with paclitaxel and cisplatin for 6 cycles and subsequently local radiotherapy. Reevaluation with chest CT showed a partial response, as indicated by the presence of a shrinking mass without any subjective symptoms. In November 2014 the patient underwent a hip bone CT which revealing osseous metastasis associated with soreness and swelling, while no histopathological examination was carried out due to intolerable pain. She subsequently received several treatment cycles (fig. 1F). During the course of treatment, the patient was periodically reviewed (fig. 1B-E, G-I). A while later, the patient accidentally discovered a pigeon egg-sized mass in her left breast which was also firm and fixed in position but not painful. In October 2016, color Doppler ultrasonography revealed a low-echo real occupancy at a distance of $4 \mathrm{~mm}$ from the skin and $5.5 \mathrm{~mm}$ from the nipple. The mass measured $19.6 \times 15.1 \times 17.3 \mathrm{~mm}$ and was located in the left upper quadrant of the breast at 1 o'clock (fig. 3A, B). Concomitantly, another low-echo real occupancy measuring $8.6 \times 4.8 \mathrm{~mm}$ (BI-RADS III, elasticity score 4) was detected (fig. 3C). A puncture biopsy was performed. Histological examination confirmed that the breast occupancy was of thymic origin according to the result of immunohistochemistry with positive expression of cytokeratin 5/6, cytokeratin19, CD5, CD117, anti-oncogene p53, whereas neuroendocrine markers (TdT, estrogen receptor, progesterone receptor, C-erbB2, GCDFP-15, GATA-3), which are highly specific for thymic carcinomas and along with the proliferation index Ki-67 have a specificity of about $90 \%$, were absent (figs. 2A, B and 4A, B). Thus, according to the clinical history and the results of relevant examinations, a final diagnosis of poorly differentiated thymic carcinoma was established, simultaneously presenting with bone and breast metastasis. Considering the poor general condition of the patient with anemia, she received symptomatic treatment such as blood transfusion and pain relief. The patient refused antineoplastic therapy.

\section{Discussion}

Primary thymic carcinoma is an epithelial tumor which occurs in the thymus characterized by malignant cytology and structure. Its biological behavior is different from common malignant thymoma. According to different cell morphology, the tumor is divided into 8 subtypes: squamous cell carcinoma, lymphoepithelioma-like carcinoma, basal cell carcinoma, mucoepidermoid carcinoma, sarcomatoid carcinoma, adenosquamous carcinoma, clear cell carcinoma, and undifferentiated carcinoma. Among them, squamous cell carcinoma and lymphoepithelioma-like carcinoma are common. The most common clinical symptoms of thymic carcinoma are chest tightness and substernal pain. Systemic manifestations include fever, fatigue, night sweats, weight loss, and ano- rexia. Due to the rapid development of thymic carcinoma lesions, most patients develop symptoms of violation of surrounding tissues as the reason for hospitalization, such as superior vena cava syndrome, coughing, bloody sputum, and hoarseness. Extrathoracic syndromes such as myasthenia gravis rarely appear with thymic carcinoma. In chest X-ray and CT examination, thymic
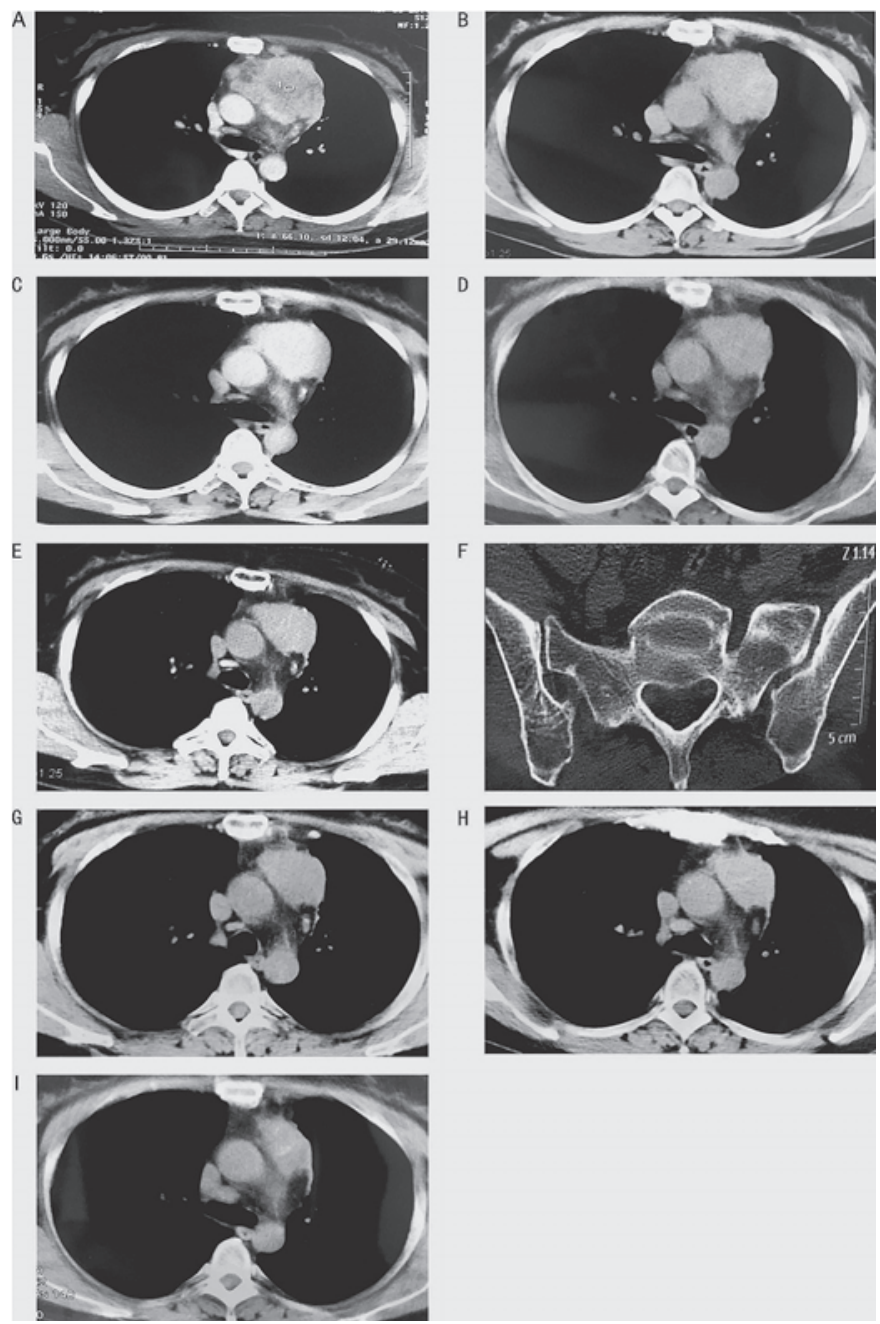

Fig. 1. A March, B April, C July, D October, and E December 2013: Tumor size gradually decreased with period chemotherapy evaluated by chest computed tomography (CT) during periodic reviews, indicating remission of the primary thymic tumor. F December 2014: Bone metastasis along with bone destruction evaluated by hip bone CT, indicating tumor progression. G May, $\mathbf{H}$ November, 2015, I April 2016: Tumor size decreased with adjusted chemotherapy evaluated by chest $\mathrm{CT}$, indicating partial tumor response.
Fig. 2. A Poorly differentiated thymic squamous cell carcinoma; histological appearance (hematoxylin staining $\times 200$ ). B Cytokeratin 5/6 in thymic carcinoma; histological appearance (hematoxylin staining $\times 200$ ).
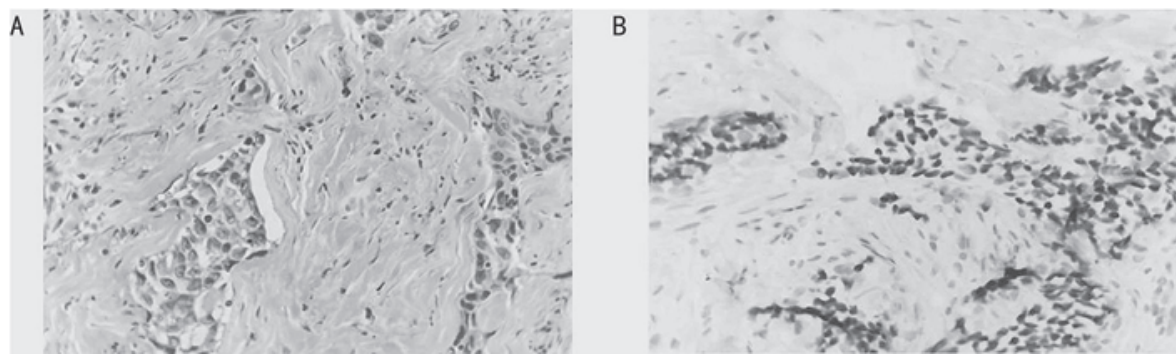
carcinoma often presents as upper anterior mediastinal swelling and is characterized by large masses clearly protruding to the side or into the bilateral pleural cavity. Density uniformity, calcification, and unclear structure with surrounding organs engulfed by masses suggest that the tumor invades adjacent organs (fig. 1A-I). Invasion is earlier and more common with thymic carcinoma, and most patients present with invasion or spread at first hospitalization. The proliferation of fibrovascular tissue during fibrosis might increase the risk of lymphovascular permeation and the possibility of recurrence and metastasis [5]. There are 3 ways thymic carcinomas can metastasize: Firstly, hematogenous metastasis; the artery supplying the thymus comes from the internal thoracic artery and the inferior thyroid artery. Secondly, lymphatic metastasis; the thymus has a specific structure different from that of other immune organs, with only efferent but no afferent lymphatics, separating it from the general lymph circulation [1]. The lymphatic vessels inject into the anterior mediastinal lymph nodes or the parasternal lymph nodes. Thirdly, direct invasion of adjacent organs [6, 7]; the tumor generally invades the surrounding organs or metastasizes and spreads to anterior mediastinal lymph nodes, the brachiocephalic vein, pleura, lung, and the pericardium. In addition to the local infiltrating growth within the mediastinum and chest, thymic carcinoma is prone to extrathoracic metastasis with the most common sites being bone, liver, and adrenal gland, but also bone marrow and brain $[8,9]$. Tumors can also metastasize to extrathoracic lymph nodes throughout the lymphatic system or implant into the pleural cavity and lung parenchyma in the chest.

Metastatic disease to the breast from non-mammary primary lesions is rather unusual. The breast has resistance to metastasis from extramammary malignancy because it contains large areas of fibrous tissue with a relatively poor blood supply [10]. According to the literature, metastasis from extramammary cancer to the breast accounts for about $0.3-2.7 \%$ of all breast malignancies [11, 12]. There are 3 sources of transfer: Firstly, hematogenous metastasis; the arterial blood supply of the breast is very rich, mainly due to intercostal perforators from the internal thoracic artery which go through the intercostal space near the sternum and then through the pectoralis major muscle and finally into the breast tissue. This accounts for about $60 \%$ of the blood supply to the breast. Secondly, lymphatic metastasis; lymph in the inner part of the breast flows through the intercostal lymphatics to the parasternal lymph nodes. Thirdly, direct invasion of adjacent organs; the most common malignant tumors metastatic to the breast include malignant melanoma, lymphoma, lung cancer, ovarian cancer, soft tis-

sue sarcoma, gastrointestinal tumors, and genitourinary cancer. There are also some reports of other tumors, such as osteosarcoma, thyroid cancer, cervical cancer, vaginal cancer, and endometrial cancer. In about $25-40 \%$ of patients with metastatic breast cancer, the initial manifestation is a breast mass [12]. Radiologically, metastatic breast tumors should be suspected when a multinodular neoplasm is found in the superficial tissue of the breast. In addition, the characteristic architectural distortion, microcalcification, and speculation associated with primary breast malignancy are uncommon in metastatic tumors [13]. Compared with primary carcinoma, the incidence rate of metastatic malignant tumors of the breast is $0.2-1.3 \%$, while it is slightly higher in postmenopausal women (up to $2-7 \%$ ) [14]. Clinically, metastatic lesions are not distinguishable from primary tumors, while radiology may be more successful in differentiating between them. In fact, in several of the cases reported, the breast lesion initially was identified and treated as a primary breast carcinoma [11]. Even in patients with a history of malignant tumors from other locations, the interval between non-mammary tumor diagnosis and development of metastatic

\section{Fig. 3. A, B}

Mass measuring $19.6 \times 15.1 \times 17.3 \mathrm{~mm}$ located in left upper quadrant of the breast at 1 o'clock. C Second low-echo real occupancy measuring $8.6 \times 4.8 \mathrm{~mm}$

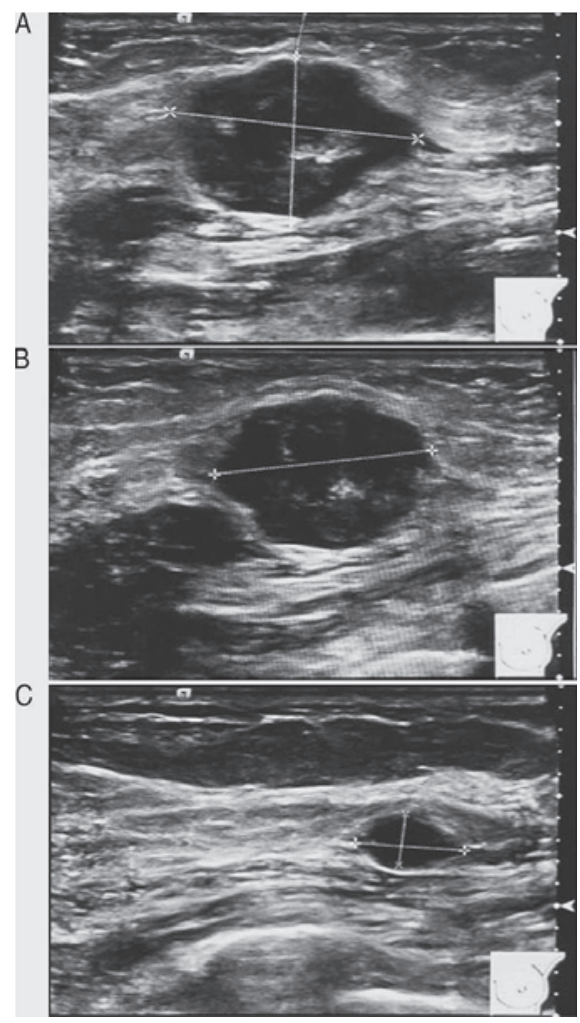

Fig. 4. A Histological appearance (hematoxylin staining $\times 200$ ): breast cancer which was confirmed to be of thymic origin. B CD117 in metastatic breast cancer; histological appearance (hematoxylin staining $\times 200$ ).

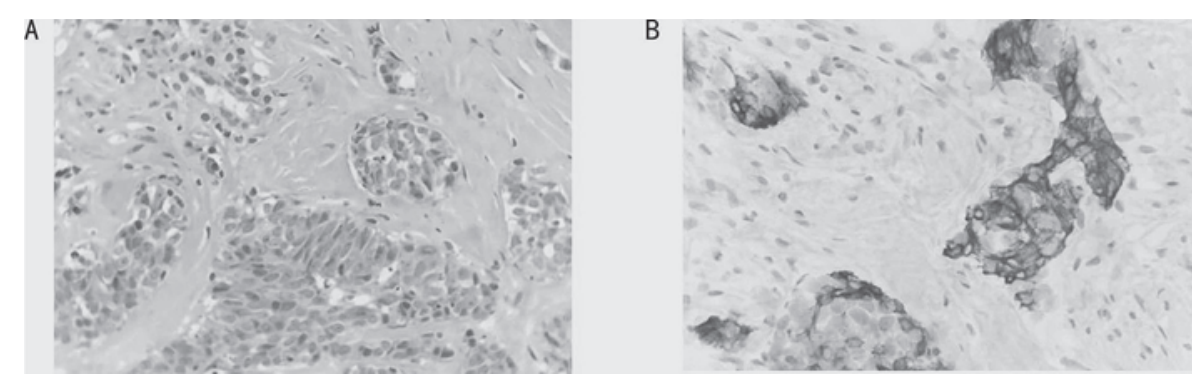

Deng/Li/Hao/Lu 
disease in the breast is relatively large and has been reported to be between 1 month and 15 years (average 1-5 years) [14].

In summary, the possibility of thymic carcinoma metastasis to the breast by lymphatic spread cannot be ruled out, but hematogenous metastasis is more likely. The breast is not the most common metastatic site for primary epithelial thymic carcinoma. However, to improve the understanding that the breast may not be protected from thymic carcinoma metastasis, regular breast examination in patients treated for thymic carcinoma is helpful in the early diagnosis of metastatic breast lesions [15]. Regular review and follow-up of patients with advanced thymic carcinoma is crucial, contributing to the detection of potential metastasis. Fine-needle aspiration cytology and core needle biopsies are the gold standard in the primary diagnosis of patients with breast neoplasms to observe changes in morphology. Immunohistochemical markers can confirm changes observed by hematoxylin \& eosin staining [16]. On balance, the disease should be detected, diagnosed, and treated early.

\section{Disclosure Statement}

There is no conflict of interest.

\section{References}

1 Moretto R, Cella CA, Raimondo L, Formisano L, Nappi L, Rescigno P, Buonerba C, Calabrese F, Ottaviano M: Tumor-to-tumor metastasis: breast cancer metastatic to thymic epithelial tumor. Anticancer Drugs 2013;24:759-764.

2 Zhao L, Zhou X, Li Z, Liu Y: Bone metastasis of malignant thymomas associated with peripheral T-cell lymphocytosis. BMC Surg 2016;16:58.

3 Kwon GY, Hong SA, Hong ME, Park ES, Yoo JH, Lee TJ: Fine needle aspiration biopsy for the diagnosis of thymoma metastatic to the breast: a case report. Acta Cytol 2010;54(suppl):798-802.

4 Chen D, Meng X, Zhao Y, Wu S: Isolated splenic metastasis from a thymic carcinoma: a case report. Cancer Biol Ther 2016;17:911-914.

5 Huang PW, Chang KM: Solitary metastasis to the breast after complete resection of encapsulated type AB thymoma: a case report. J Med Case Rep 2015;9:63.

6 Nagamata M, Okuma Y, Hosomi Y, Hishima T: Thymic carcinoma with endobronchial metastasis: a case report. J Bronchology Interv Pulmonol 2017;24: 159-162.
7 Taira N, Kawabata T, Furugen T, Ichi T, Kushi K, Yohena T, Kawasaki H, Ishikawa K: Repeated localized treatment for endobronchial metastasis of thymic carcinoma. Am J Case Rep 2015;16:483-485.

8 Sharma S, Dawson L: A rare tumor with a very rare initial presentation: thymic carcinoma as bone marrow metastasis. Case Rep Pathol 2017;2017:6497376.

9 Kosty JA, Andaluz N: Metastatic thymic carcinoma presenting as a posterior fossa mass: case report and review of the literature. World Neurosurg 2016;93:486. e1-6.

10 Kim SM, Ko EY, Han BK, Shin JH, Kang SS, Nam SJ, Cho EY: Metastatic thymoma of the breast. Korean J Radiol 2008;9:80-83.

11 Georgiannos SN, Aleong JC, Goode AW, Sheaff M: Secondary neoplasms of the breast: a survey of the 20th century. Cancer 2001;92:2259-2266.

12 Tang F, Jiang GY, Wang NX: Metastasis to breast of gastric cancer: a case report and literature review (Article in Chinese). Chin J Breast Dis 2012;6:708-712.
13 Pai T, Nair N, Pantvaidya G, Deodhar K, Shet T: Metastatic nasopharyngeal carcinoma presenting as an isolated breast mass: a diagnostic pitfall and a review of literature. Indian J Pathol Microbiol 2017;60:119-121

14 Alvarado CI, Carrera AM, Perez MD, Tavassoli FA: Metastases to the breast. Eur J Surg Oncol 2003;29: 854-855.

15 Haen SP, Stroebel P, Marx A, Suesskind D, Fend F, Reichmann U, Kopp HG, Kanz L, Mayer F: Choroidal metastases from thymic carcinoma during pregnancy: case report. BMC Cancer 2015;15:972.

16 Leisibach P, Schneiter D, Soltermann A, Yamada Y, Weder W, Jungraithmayr W: Prognostic value of immunohistochemical markers in malignant thymic epithelial tumors. J Thorac Dis 2016;8:2580-2591. 